\title{
Thelper cell IL-4 drives intestinal Th2 priming to oral peanut antigen, under the control of OX4OL and independent of innate-like lymphocytes
}

DK Chu ${ }^{1}$, Z Mohammed-Ali ${ }^{1}$, R Jiménez-Saiz ${ }^{1}$, TD Walker ${ }^{1}$, S Goncharova ${ }^{1}$, A Llop-Guevara ${ }^{1}$, J Kong ${ }^{1}$, ME Gordon ${ }^{1}$, NG Barra ${ }^{1}$, AE Gillgrass ${ }^{1}$, H Van Seggelen ${ }^{1}$, WI Khan ${ }^{2}$, AA Ashkar ${ }^{1}$, JL Bramson ${ }^{1}$, AA Humbles ${ }^{3}$, R Kolbeck $^{3}, S$ Waserman ${ }^{4}$ and M Jordana ${ }^{1}$

Intestinal T helper type 2 (Th2) immunity in food allergy results in IgG1 and IgE production, and antigen re-exposure elicits responses such as anaphylaxis and eosinophilic inflammation. Although interleukin-4 (IL-4) is critically required for allergic sensitization, the source and control of IL-4 during the initiation of Th2 immunity in vivo remains unclear. Nonintestinal and non-food allergy systems have suggested that natural killer-like T (NKT) or $\gamma \delta$ T-cell innate lymphocytes can supply the IL-4 required to induce Th2 polarization. Group 2 innate lymphoid cells (ILCs) are a novel IL-4-competent population, but their contribution to initiating adaptive Th2 immunity is unclear. There are also reports of IL-4-

independent Th2 responses. Here, we show that IL-4-dependent peanut allergic Th2 responses are completely intact in NKT-deficient, $\gamma \delta$ T-deficient or ILC-deficient mice, including antigen-specific IgG1/lgE production, anaphylaxis, and cytokine production. Instead, IL-4 solely from $\mathrm{CD} 4{ }^{+}$Th cells induces full Th2 immunity. Further, CD4 ${ }^{+}$Th cell production of IL-4 in vivo is dependent on OX40L, a costimulatory molecule on dendritic cells (DCs) required for intestinal allergic priming. However, both Th2 cells and ILCs orchestrated IL-13-dependent eosinophilic inflammation. Thus, intestinal Th2 priming is initiated by an autocrine/paracrine acting CD4 ${ }^{+}$Th cell-intrinsic IL-4 program that is controlled by DC OX $40 \mathrm{~L}$, and not by NKT, $\gamma \delta \mathrm{T}$, or ILC cells.

\section{INTRODUCTION}

Allergies are detrimental immune responses to specific environmental antigens that are normally tolerated by most individuals. Food allergies affect $2-10 \%$ of the general population, causing symptoms ranging from pruritus and urticaria to systemic, potentially life-threatening reactions, called anaphylaxis. ${ }^{1,2}$ Peanut (PN) allergy is highly associated with anaphylaxis. ${ }^{3}$ Central to the pathogenesis of allergies are $\mathrm{T}$ helper type 2 (Th2) cells, which are characterized by their production of interleukin-4 (IL-4), IL-5, and IL-13, provision of $B$ cell help in isotype switching to IgG1 and IgE, and orchestration of eosinophilic inflammation within allergen re-exposed tissues. An important focus of current research is to investigate the mechanisms underlying allergic sensitization, i.e., Th2 generation.

Of the signature Th2 cytokines, IL- 4 has been reported to be critically required to mount full Th2 responses, understood as isotype switching to IgE and generation of Th2 cells. IL-4 and T-cell receptor (TCR) activation alone can promote the generation of Th2 cells in vitro. However, the source of IL-4 that initiates the development of naïve $\mathrm{CD}^{+}{ }^{+} \mathrm{T}$ cells into IL-4 expressing Th2 cells in vivo remains to be clarified ${ }^{4-6}$ and is unknown in food allergy.

A prominent hypothesis is that naïve $\mathrm{T}$ cells receive IL-4 from an innate cell capable of rapid IL-4 production such as mast cells, basophils, or innate-like lymphocytes including $\gamma \delta$

\footnotetext{
${ }^{1}$ Departments of Pathology and Molecular Medicine, McMaster Immunology Research Centre, McMaster University, Hamilton, Ontario, Canada. ${ }^{2}$ Departments of Pathology and Molecular Medicine, Farncombe Family Digestive Health Research Institute, McMaster University, Hamilton, Ontario, Canada. ${ }^{3}$ Departments of Respiratory, Inflammation and Autoimmunity, Medlmmune LLC, Gaithersburg, Maryland, USA and ${ }^{4}$ Department of Medicine, McMaster University, Hamilton, Ontario, Canada. Correspondence: M Jordana (jordanam@mcmaster.ca)
} 
T cells, natural killer-like T (NKT) cells, or group 2 innate lymphoid cells (ILC2s). The latter are a novel population of IL-4 competent cells that have a critical role in effecting type 2 immune responses through IL- 5 and IL- 13 production, but their contribution to initiating Th2 immunity is unclear. $^{7}$

$\gamma \delta \mathrm{T}$ cells develop in the thymus from double negative thymocytes undergoing TCR $\beta, \gamma$, and $\delta$ gene rearrangement; deficiency of TCR $\delta$ or TCR $\gamma$ blocks $\gamma \delta$ T-cell development. Following TCR rearrangement, $\gamma \delta$ T cells preferentially localize to epithelial sites at barrier surfaces such as the lung and the intestine. There, they express a limited TCR repertoire and when activated, rapidly produce a number of effector molecules, including IL-4. ${ }^{8}$ Indeed, in a model of allergic asthma, where sensitization is achieved through the intraperitoneal route, immunized $\gamma \delta \mathrm{T}$ cell-deficient mice showed decreased levels of serum ovalbumin-specific IgE and IgG1 antibodies, which were restored with IL-4 administration. ${ }^{9}$ Hence, it has been suggested that $\gamma \delta \mathrm{T}$ cells may be a critically required source of IL-4 for the development of Th2 responses.

NKT cells develop in the thymus from double positive thymocytes, are classically defined as $\mathrm{CD}^{+}{ }^{+} \mathrm{NK} 1.1^{+}$or $\mathrm{TCR} \beta^{+} \mathrm{NK} 1.1^{+}$cells and are another innate-like lymphocyte capable of rapidly producing IL-4 upon activation. ${ }^{10}$ Development, maturation and survival of NKT cells critically depends on recognition of CD1d- $\beta 2$ microglobulin $(\beta 2 \mathrm{~m})$ complexes, as well as IL-15. ${ }^{11,12}$ Hence, loss of CD1, $\beta 2 \mathrm{~m}$, or IL15 results in a severe lack of NKT cells. The TCR of NKT cells recognizes mainly lipid antigens, and this property is taken advantage of when quantifying NKT cells by reactivity to $\alpha$ galactosylceramide ( $\alpha$ GalCer)-loaded CD1d tetramers. NKT cells have been proposed to regulate allergic asthma development via their production of type 2 cytokines such as IL-4 $4^{13,14}$ and lipids within foods have been proposed to be one potential source of NKT cell activation in food allergy. ${ }^{15}$ However, the contribution of NKT cells to the development of food allergy remains unknown.

An alternative model of Th2 differentiation states that naïve Th cells do not require IL- 4 to be programmed to become Th2 cells. Instead, costimulatory molecules such as OX40L and Jagged2 have been suggested to drive Th2 response development. ${ }^{5,6,16}$ Here, we used an established model of PN allergy ${ }^{17-20}$ to investigate the cellular source and regulation of IL-4 during the initiation of type 2 immunity to oral antigen. We show that neither $\gamma \delta$ T nor NKT cell deficiency impacts the development or expression of Th2 immunity to PN. Similarly, ILC deficiency does not impair Th2 priming, Ig production, or clinical anaphylaxis, but does impair eosinophilic inflammatory responses due to reduced IL-13 and IL- 5 production. Instead, IL-4 production solely from CD $4^{+}$Th cells, under the control of OX40L, allows for full anaphylactic PN allergic Th2 responses to develop. Hence, rather than dichotomous costimulation vs. IL-4 signaling-dependent Th2 generation, we show that in vivo development of Th2 cells to a common food allergen follows a pathway requiring both costimulation and IL-4 from $\mathrm{CD}^{+}{ }^{+} \mathrm{T}$ cells, independent of innate lymphocytes.

\section{RESULTS}

\section{IL-4 is required for PN allergy}

We previously showed that weekly gavage of PN and the adjuvant, cholera toxin (CT), for 4 weeks induces IL-4
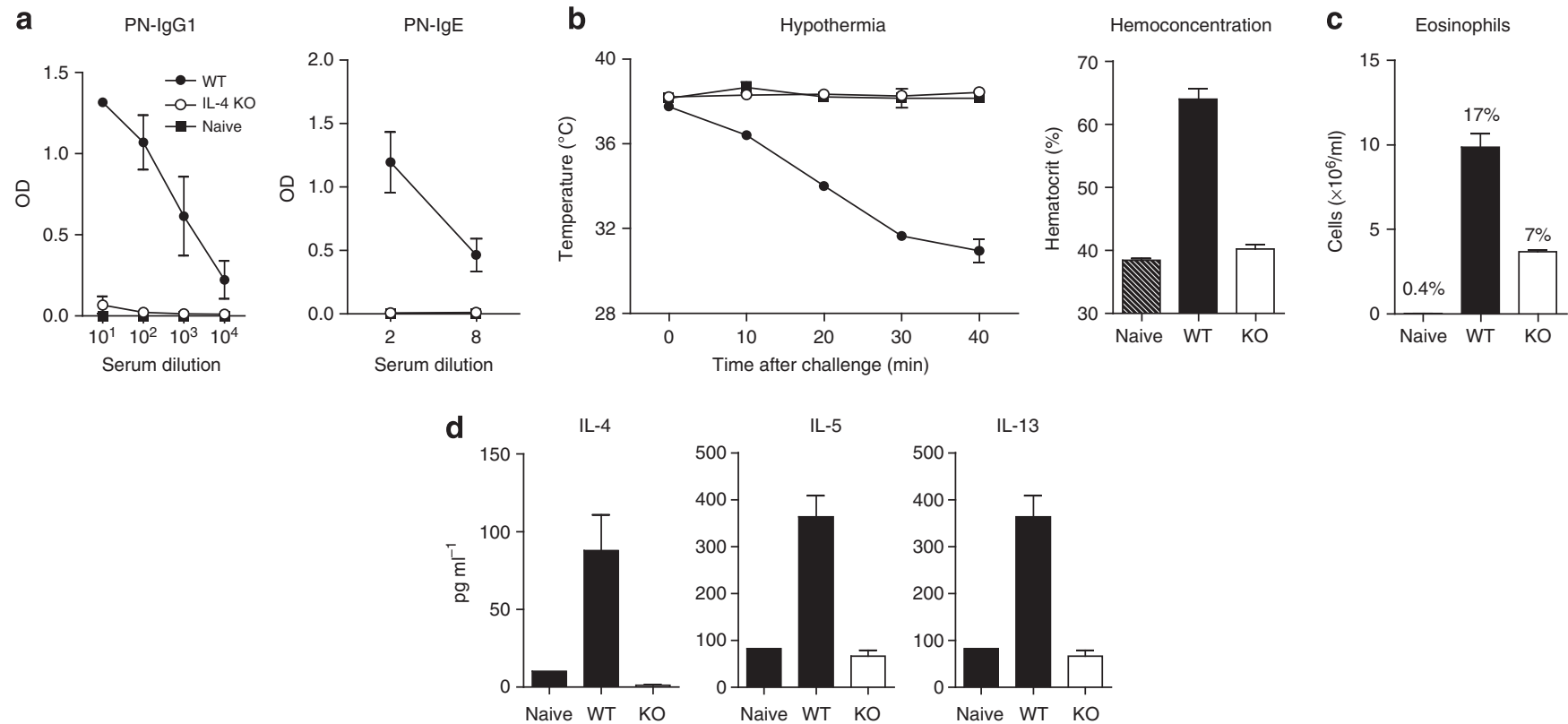

Figure 1 Interleukin-4 (IL-4) is required for peanut (PN) allergy. Wild-type (WT) or IL-4 knockout (KO) mice were exposed to PN + CT intragastrically (i.g.) weekly for 4 weeks, or kept naive. (a) Serum PN-specific-IgG1 (PN-IgG1, left) and -lgE (right) at week 5. (b) Anaphylactic hypothermia (left) and hemoconcentration at 40' (right) after intraperitoneal (i.p.) PN challenge at week 5. (c) Peritoneal eosinophilia recovered $72 \mathrm{~h}$ after challenge. (d) T helper type 2 (Th2) cytokine production from PN-stimulated splenocytes. Mean \pm s.e.m., $n=3-5 /$ group/experiment, two pooled experiments. CT, cholera toxin. 

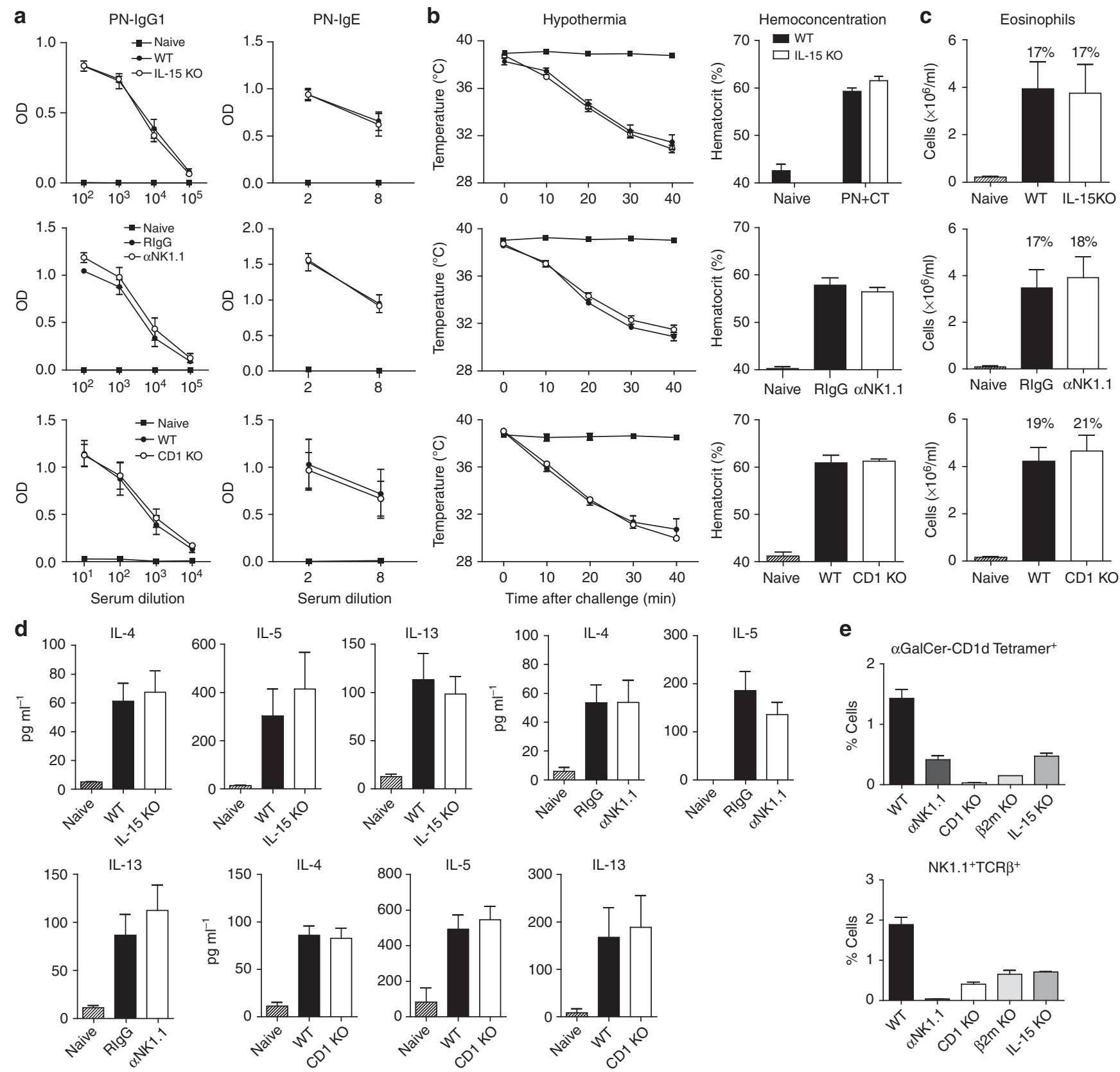

Figure 2 Interleukin-15 (IL-15), CD1, or NK1.1 $1^{+}$cells are not required to induce food allergy and anaphylaxis to peanut (PN). Wild-type (WT) mice receiving rat IgG (RlgG) or anti-NK1.1 ( $\alpha$ NK1.1) (middle row), or CD1 knockout (KO) (bottom row) or IL-15 KO mice (top row) were exposed to PN + CT intragastrically (i.g.) weekly for 4 weeks, or kept naïve. (a) Serum PN-lgG1 (left) and PN-IgE (right). (b) Anaphylactic hypothermia (left) and hemoconcentration (right). (c) Peritoneal eosinophilia. (d) Thelper type 2 (Th2) cytokine production from PN-stimulated splenocytes. (e) Natural killer-like $\mathrm{T}(\mathrm{NKT})$ cell quantification by CD19- ${ }^{-} \alpha \mathrm{GalCer-CD1d}$ tetramer ${ }^{+}$or NK1.1 $1^{+} \mathrm{TCR} \beta^{+}$staining in mesenteric lymph nodes (MLNs) by flow cytometry. Mean \pm s.e.m., $n=3-8 /$ group/experiment, 2-3 pooled experiments. CT, cholera toxin; $\alpha$ GalCer, $\alpha$-galactosylceramide; TCR, T-cell receptor.

dependent PN-specific IgG1 production by week 5 and establishes, at that time, susceptibility to undergo anaphylaxis upon intraperitoneal PN challenge. ${ }^{17}$ Here, we confirm these data, and further show that PN-specific IgE, inflammatory, and Th2 cytokine responses are also dramatically impaired in IL-4 deficient (IL-4 knockout (KO)) mice (Figure 1). These data show that $\mathrm{T}$ and $\mathrm{B}$-cell allergic responses to oral antigen require IL-4.

\section{NKT cells are not required for Th2 responses to PN}

We assessed the requirement for NKT cells during allergic sensitization to PN by four methods: IL- $15, \beta 2 \mathrm{~m}$, or CD1 KO mice, or by depletion with anti-NK1.1 antibody in wild-type (WT) mice. NKT depletion was assessed by $\alpha$ GalCer-loaded CD1d tetramer reactivity as well as classical NK1.1 ${ }^{+} \mathrm{TCR}^{+}{ }^{+}$costaining (Figure 2e). Compared with PN-sensitized WT mice, IL-15 KO mice generated comparable PN-specific IgG1 and IgE 

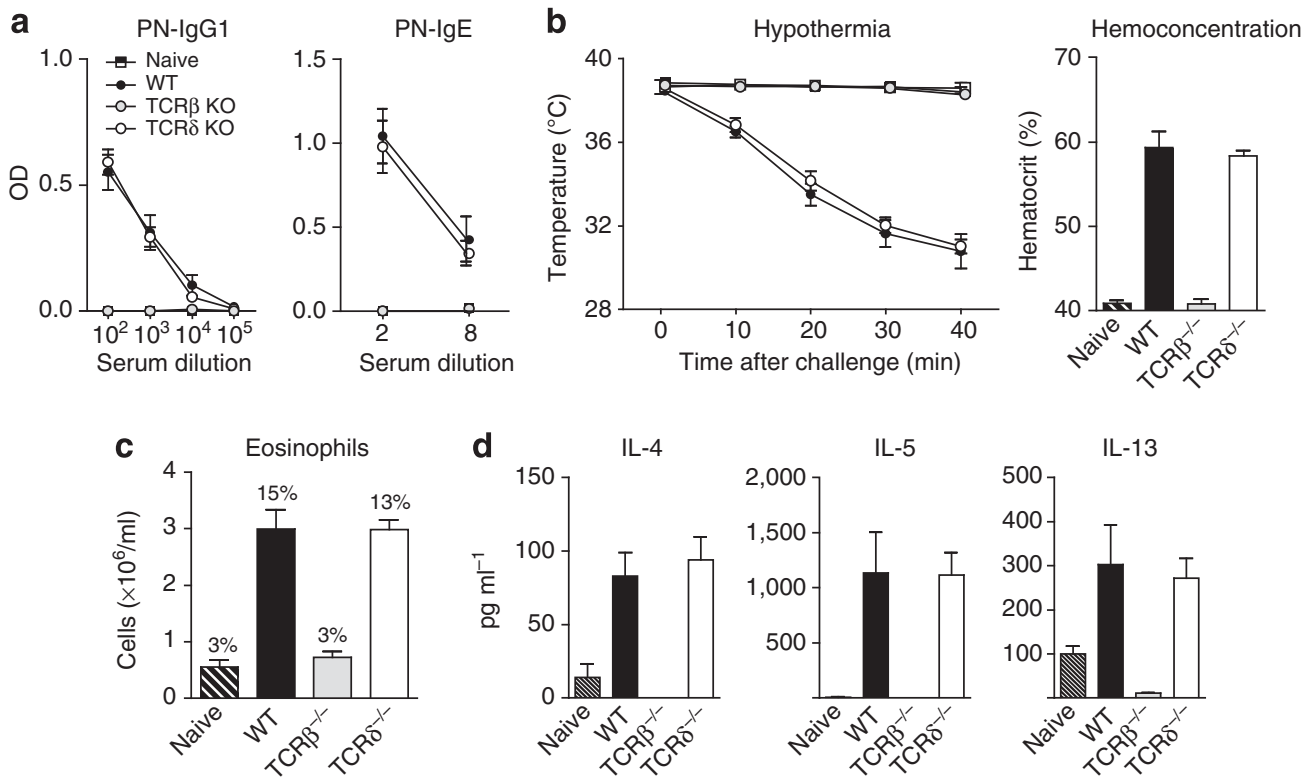

Figure $3 \gamma \delta$ T cells do not regulate food allergy and anaphylaxis to peanut (PN). Wild-type (WT), TCR $\delta$, or TCR $\beta$ knockout (KO) mice were exposed to PN + CT intragastrically (i.g.) weekly for 4 weeks, or kept naïve. (a) Serum PN-lgG1 (left) and PN-lgE (right). (b) Anaphylactic hypothermia (left) and hemoconcentration (right). (c) Peritoneal eosinophilia. (d) T helper type 2 (Th2) cytokine production from PN-stimulated splenocytes. Mean \pm s.e.m., $n=3-8 /$ group/experiment, $2-3$ pooled experiments. CT, cholera toxin; TCR, T-cell receptor.

titers (Figure 2a, top row). Clinically, this led to comparable hypothermia and hemoconcentration anaphylactic responses (Figure $\mathbf{2 b}$, top row) in both sensitized groups. Peritoneal eosinophilic inflammation (Figure 2c, top row) was similarly significantly elevated over naïve levels in both challenged strains. Th2 cytokine production by both sensitized groups was similarly elevated above naïve levels (Figure 2d). Similar results were generated with anti-NK1.1 treated mice (Figure 2a-d, middle row). We also found similar results with $\beta 2 \mathrm{~m}$ KO mice (Supplementary Figure S1 online), as well as CD1 KO mice (Figure 2a-d, bottom row). These data collectively show that Th2 responses to PN develop largely unimpaired in the absence of NKT cells as a result of deficiency in IL-15, CD1, $\beta 2 \mathrm{~m}$, or NK1.1 ${ }^{+}$cells.

\section{$\gamma \delta \mathrm{T}$ cells do not regulate allergic responses to $\mathrm{PN}$}

To investigate the role of $\gamma \delta$ T cells in the development of PN allergic Th2 immunity, WT or TCR $\delta$ KO mice were sensitized to $\mathrm{PN}$ and evaluated for subsequent $\mathrm{T}$ - and $\mathrm{B}$-cell responses. $\mathrm{PN}$-sensitized WT and TCR $\delta \mathrm{KO}$ mice generated similar $\mathrm{PN}$-specific IgG1 and IgE (Figure 3a) titers, resulting in clinically indistinguishable hypothermic and hemoconcentration anaphylactic responses upon challenge (Figure 3b). Similarly, both sensitized groups developed comparably robust eosinophilic peritoneal inflammation (Figure 3c) and Th2 cytokine responses (Figure 3d). In contrast, TCR $\beta$ KO mice exhibited none of these features (Figure 3). Similar results to the TCR $\delta$ KO were generated when treating mice throughout sensitization with anti- $\gamma \delta$ TCR antibody clone UC7-13D5 (Supplementary Figure S2). These data show that $\gamma \delta$ T cells do not significantly regulate the initiation of $\mathrm{Th} 2$ responses to $\mathrm{PN}$.

\section{$\mathrm{CD}^{+}{ }^{+} \mathrm{T}$ cell-intrinsic IL-4 drives sensitization to PN}

To further explore the source of IL-4 responsible for the initiation of PN-induced Th2 immunity, we restricted IL-4 expression to $\mathrm{CD} 4^{+} \mathrm{T}$ cells by sublethally irradiating CD $45.2^{+}$ WT or IL-4 KO mice and, adoptively transferring purified congenic CD45.1 ${ }^{+}$IL-4-competent CD $4^{+}$Th cells to these hosts (Figure 4a; Supplementary Figure S3a). Thus, the sole source of IL- 4 in recipient IL- 4 KO mice is from CD45.1 marked, $\mathrm{CD} 4{ }^{+}$Th cells that have preserved endogenous $\mathrm{T}$ cell (CD45.2) competition for homeostatic/activation signals. Under these conditions, $60-80 \%$ of the recipient's $\mathrm{CD}^{+}$ Th cell compartment were made up of CD $45.1^{+} i l 4^{+/+} \mathrm{T}$ cells (Figure 4b; Supplementary Figure S3b). No abnormalities in overall $\mathrm{CD} 4^{+}$T-cell frequency or activation status in either $\mathrm{CD} 45.1^{+}$or $\mathrm{CD} 45.2^{+}$T-cell pools were observed (Supplementary Figure S3b). Compared with control CD45.1 $\rightarrow$ WT CD45.2 chimeras sensitized to $\mathrm{PN}(\mathrm{WT}+\mathrm{T})$, $\mathrm{CD} 45.1 \rightarrow \mathrm{IL}-4 \mathrm{KO}$ CD45.2 chimeras $(\mathrm{KO}+\mathrm{T})$ generated comparable levels of antigen-specific IgG1 (Figure 4c) and IgE (Figure 4d), and upon challenge, anaphylactic responses (Figure 4e). Eosinophilic inflammation $72 \mathrm{~h}$ after challenge was also similarly elevated (Figure $\mathbf{4 f}$ ), as was cytokine production (Figure 4g). These data show that in vivo, IL-4 production solely from $\mathrm{CD} 4{ }^{+} \mathrm{T}$ cells allows for full development of Th2 responses to PN. Conversely, this also shows that non-CD4 ${ }^{+}$sources of IL- 4 are dispensable during the development of Th2 immunity in this system.

\section{OX40L controls T cell-intrinsic IL-4 production during PN sensitization}

We have previously shown that OX40L, expressed on dendritic cells (DCs), is required to initiate $\mathrm{PN}$ allergic Th2 responses. ${ }^{17}$ 
a

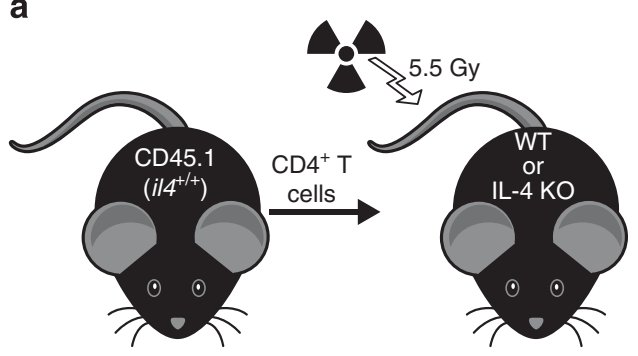

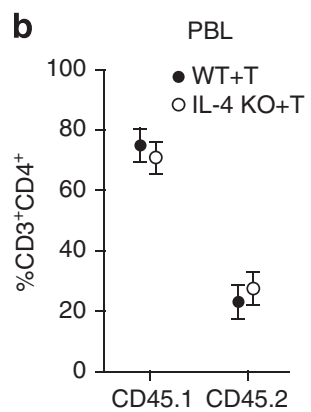
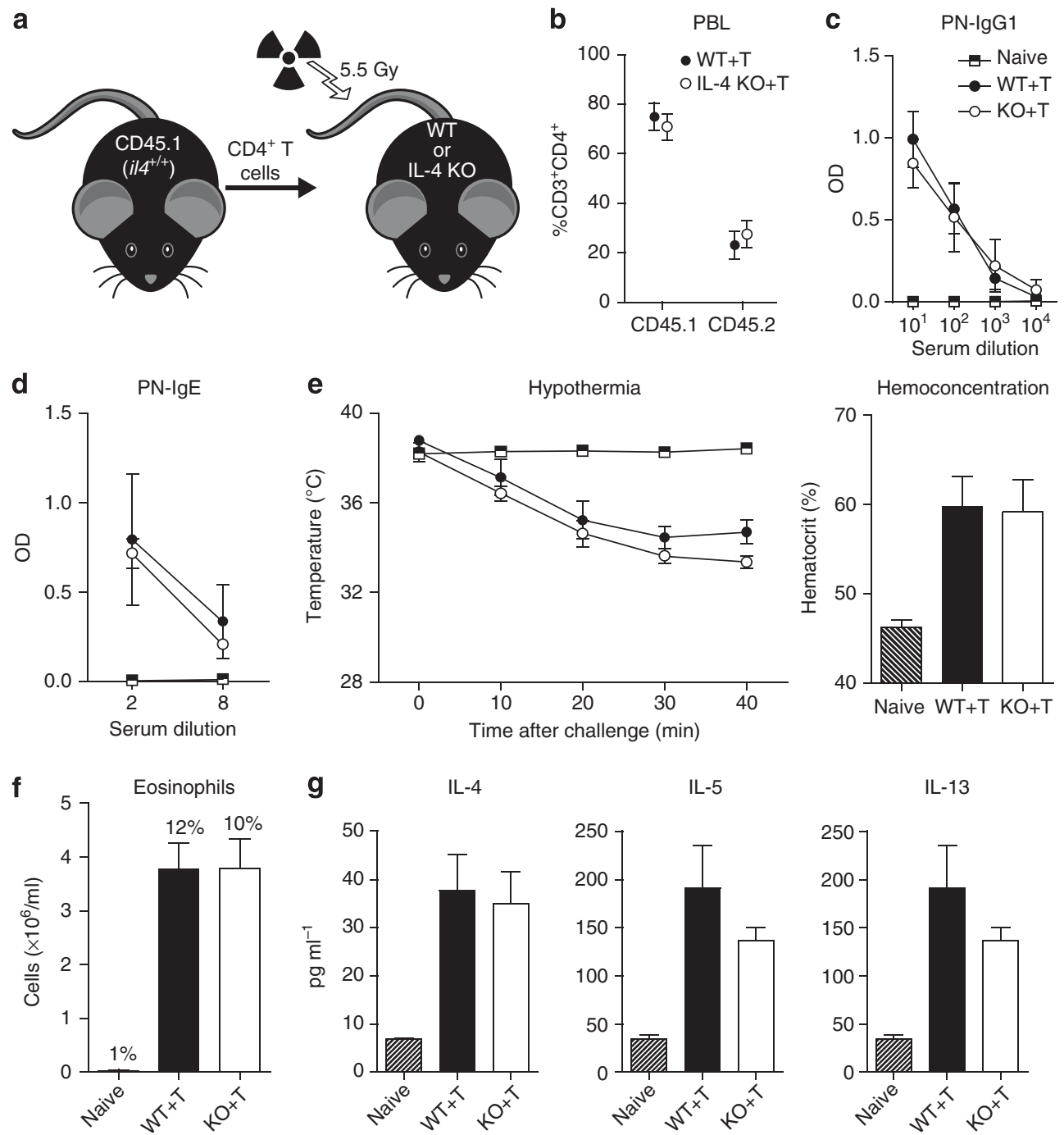

Figure $4 \mathrm{CD}^{+}$T cell-intrinsic interleukin-4 (IL-4) drives peanut (PN) allergy and anaphylaxis. (a) Transfer of $i 4^{+/+} \mathrm{CD}^{+} 5.1^{+} \mathrm{CD} 4{ }^{+} \mathrm{T}$ cells to sublethally irradiated CD45.2+ $2^{+}$wild-type (WT) (WT + T) or IL-4 knockout (KO) recipients (IL-4 KO + T). (b) Peripheral blood (PBL) T helper cell composition at week 0 of PN sensitization. Mice were exposed to PN + CT intragastrically (i.g.) weekly for 4 weeks, or kept naïve. (c) Serum PN-IgG1 or (d) PN-IgE at week 5. (e) Anaphylactic hypothermia (left) and hemoconcentration (right). (f) Peritoneal eosinophilia. (g) T helper type 2 (Th2) cytokine production from PN-stimulated splenocytes. Mean \pm s.e.m., $n=2-7 /$ group/experiment, two pooled experiments. CT, cholera toxin.

To test whether this requirement is due to control of Th cellintrinsic IL-4 production in vivo, we used 4get il4 reporter mice, which express eGFP from a bicistronic locus shared with $i l 4{ }^{21}$ These mice were exposed to $\mathrm{PN}+\mathrm{CT}$, with or without neutralizing anti-OX40L antibody, and then draining mesenteric lymph nodes (LNs) were analyzed for il4 expression, marked by eGFP. PN + CT exposure resulted in an approximate doubling of the percentage of $\mathrm{IL}-4 \mathrm{eGFP}^{+} \mathrm{CD} 3{ }^{+} \mathrm{CD} 4{ }^{+} \mathrm{T}$ cells, which was prevented with OX40L neutralization (Figure 5a). Gating and quantification of this population showed that $\gamma \delta \mathrm{T}$ cells and $\mathrm{CD}_{4} 9 \mathrm{~b}^{+}$NKT cells made up a minority $(<2 \%)$ of this population (Figure 5a), and that neither changed in percentage or number during Th2 priming to $\mathrm{PN}$, or OX40L blockade (Figure $\mathbf{5 b}, \mathbf{c}$ ). In contrast, $\mathrm{CD} 49 \mathrm{~b}^{-} \mathrm{TCR} \gamma \delta^{-}$Th2 cells markedly expanded approximately threefold after PN sensitization, which was inhibited by OX40L blockade (Figure 5d). Hence, IL-4 production during $\mathrm{PN}$ sensitization is primarily from conventional Th cells and is under the control of OX40L.

\section{ILC depletion impacts eosinophilic inflammation, but not Th2 initiation}

ILC2s are a novel population of non- $\mathrm{B}$, non-T cells expressing c-Kit, Sca-1, IL-7R $\alpha$, ST2, CD25, CD44, and CD90 (Thy1) that have a critical role in effector type inflammatory responses to allergens and helminths. ${ }^{7}$ They are potent sources of IL-5 and IL-13, but have also been found to be IL- 4 competent and to be present within LNs, ${ }^{17,22,23}$ but their contribution to regulating adaptive Th2 immunity remains unclear. We previously observed expansion of ILCs during PN sensitization. ${ }^{17}$ 
a
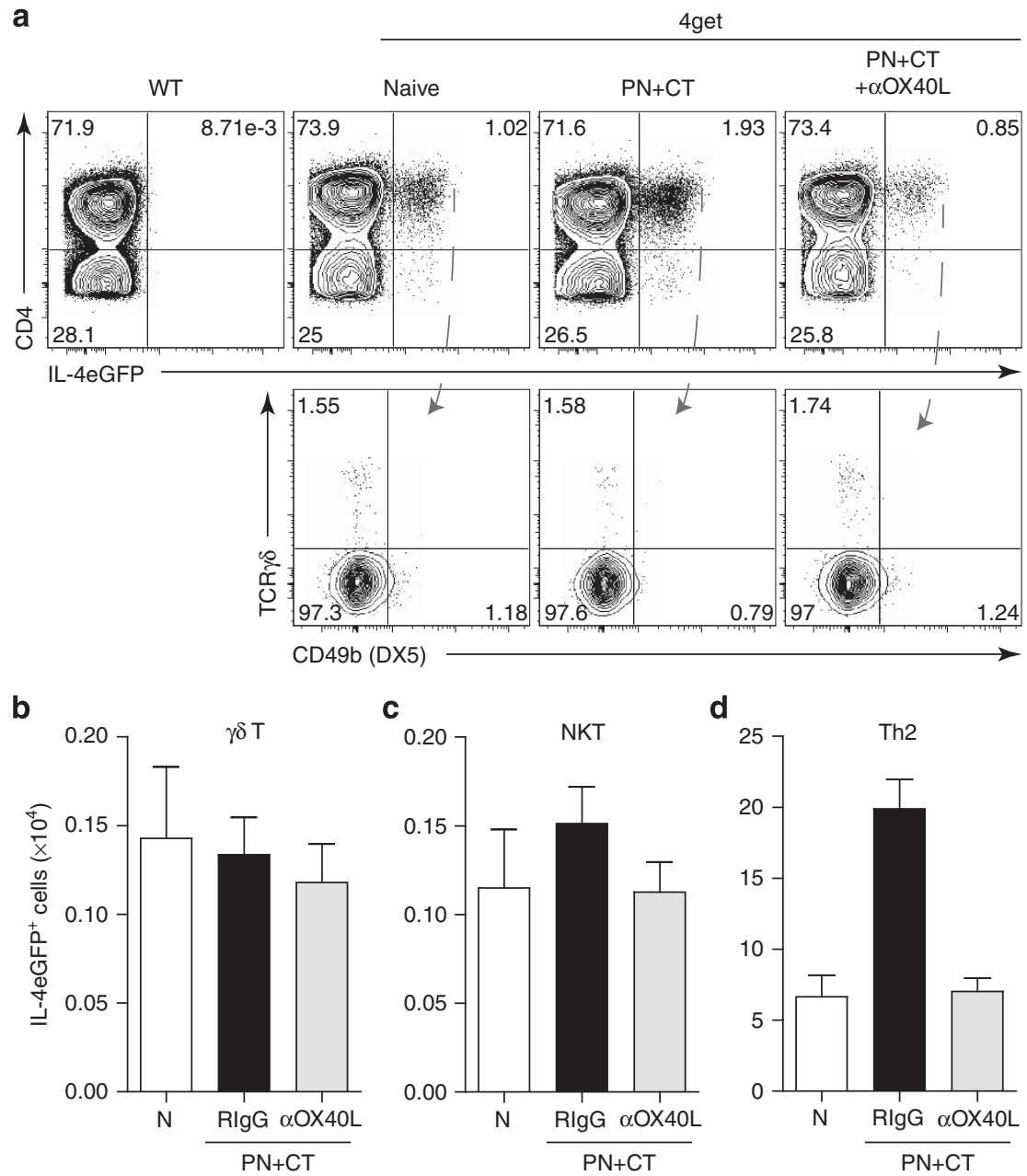

Figure 5 Interleukin-4 (IL-4) originates from conventional Th cells during peanut (PN) sensitization, and IL-4 production is controlled by OX40L in vivo. Mice were exposed to PN + CT intragastrically (i.g.) with or without anti-OX40L administration and then mesenteric lymph nodes (MLNs) were evaluated $96 \mathrm{~h}$ later, or kept naïve $(\mathrm{N})$. (a) Representative flow cytometry plots after PN sensitization with or without OX40L neutralization. Quantification of IL-4

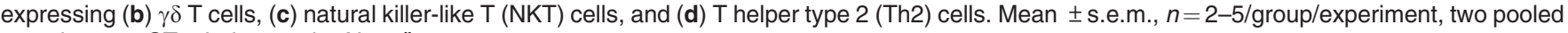
experiments. CT, cholera toxin; N, naïve.

Consistent with this, after $\mathrm{PN}+\mathrm{CT}$ priming we observed a minor expansion of IL- $4^{+}$non-B/T cells, which were mainly ILC2s, as evidenced by their IL-4 competency and phenotypic expression of CD44, CD25, ST2, Thy1, c-Kit IL-7R $\alpha$, and Sca-1 (Supplementary Figure S4a). Hence, we next evaluated whether ILC2s contributed to Th2 priming. To accomplish this, we generated CD90-disparate chimeras (Supplementary Figure S4b), which allow for antibody-mediated depletion of CD90.2 $2^{+}$ILCs, but not CD90.1 ${ }^{+}$Th cells. ${ }^{24}$ ILC2 depletion (Figure 6a) was not accompanied by any impact on PN-IgG1/ IgE production, anaphylactic responses, or splenocyte recall Th2 cytokine production (Figure $\mathbf{6 b}-\mathbf{d}$ ). However, ILC2 depletion significantly impaired post-anaphylaxis allergic eosinophilic inflammatory responses (Figure 6e). Consistent with the potent IL- 5 and IL-13 producing ability of ILC2s, ${ }^{23,25}$ anti-CD90.2-treated mice exhibited reduced levels of IL-5 and IL-13 within their peritoneal cavities (Figure 6f). To explore the contribution of Lineage ${ }^{-} \mathrm{IL}-7 \mathrm{R} \alpha^{+} \mathrm{Sca}-1^{+} \mathrm{ST} 2{ }^{+} \mathrm{CD} 90^{+}$ ILC2s and $\mathrm{CD} 3{ }^{+} \mathrm{CD} 4{ }^{+}$Th cells to IL-13 and IL-5 production during peritoneal allergic inflammation, we performed intracellular cytokine staining and found that ILC2s and Th cells produced IL-5 and IL-13 in an approximate 1:2 ratio (Figure 6g). In agreement with an important role for ILC2-derived IL-13 in driving eosinophilic inflammation, we found that IL-13 KO mice mounted markedly impaired eosinophilic inflammatory responses (Figure 6h). Notably, similar to ILC2-deficient mice, IL-13 KO mice do not exhibit a defect in Th priming, Ig production, or anaphylaxis to $\mathrm{PN}^{17}$ (Supplementary Figure S4c). Thus, although ILC2s have an important role in effecting type 2 immunity within antigen-exposed tissues through IL-13 and IL-5 production, they do not regulate the initiation of adaptive Th2 immunity in this system.

\section{DISCUSSION}

Th2 induction to oral antigen causes food allergy, a growing health problem with potentially fatal consequences. Although IL-4 is often thought to be critical for the development of Th2 

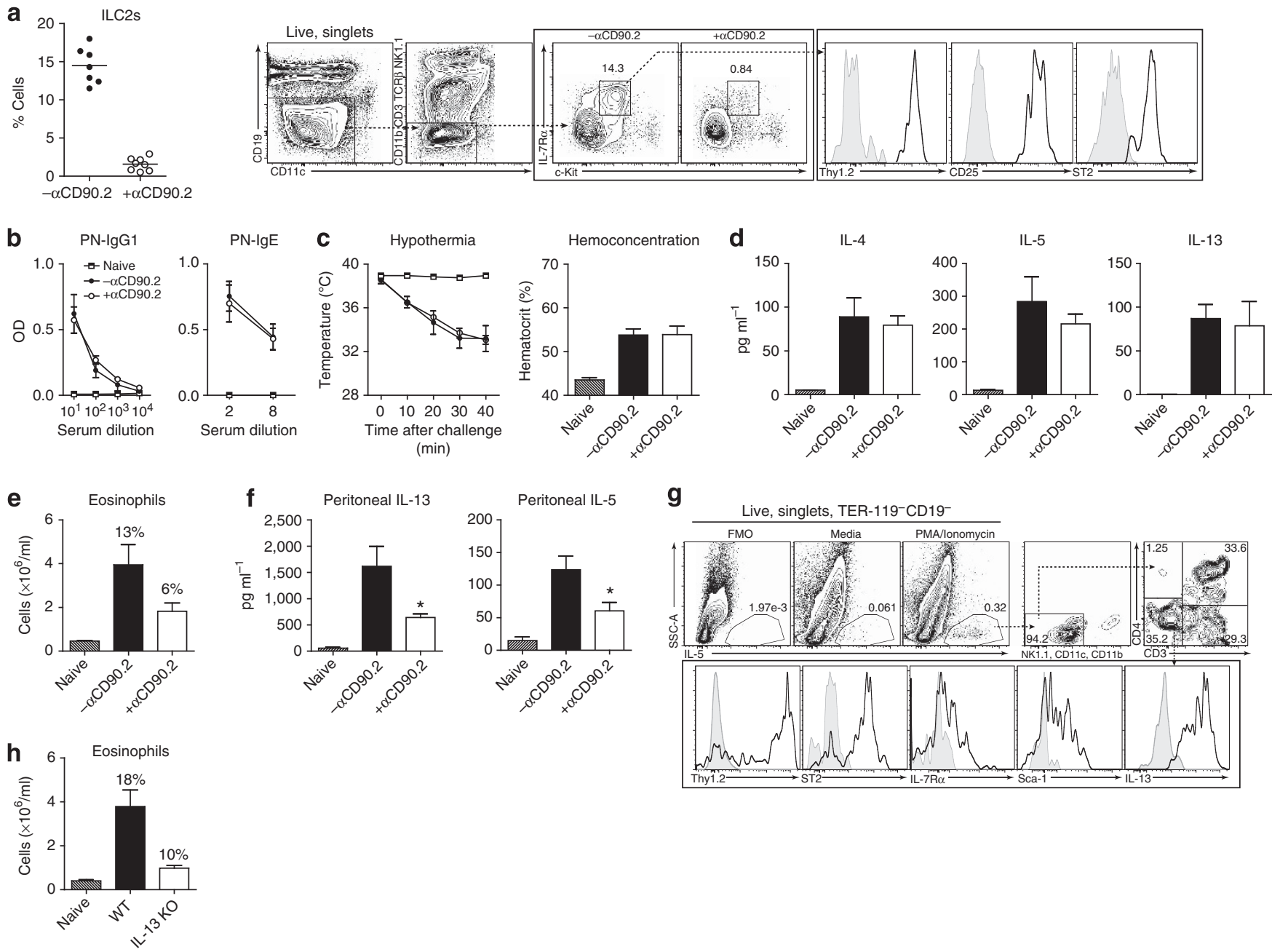

Figure 6 Innate lymphoid cell (ILC) depletion prevents allergic inflammation but not sensitization. CD90-disparate chimeras were generated by transferring CD90.1 ${ }^{+}$Th cells to CD90.2 ${ }^{+}$TCR $\beta$ knockout (KO) mice and then they were treated with anti-CD90.2 antibody throughout sensitization (peanut (PN) + CT intragastrically (i.g.) weekly for 4 weeks) and challenge to PN, or kept naïve. (a) ILC2 depletion and gating strategy in spleen. (b) Serum PN-IgG1 (left) and PN-IgE (right) at week 5. (c) Anaphylactic hypothermia (left) and hemoconcentration (right). (d) T helper type 2 (Th2) cytokine production from PN-stimulated splenocytes. (e) Peritoneal eosinophilia. (f) Peritoneal lavage interleukin-13 (IL-13) and IL-5 $72 \mathrm{~h}$ after challenge. (g) Flow cytometric analysis of IL-5 and IL-13 producing cells in peritoneal inflammatory infiltrates. (h) Peritoneal eosinophilia in sensitized and challenged wildtype (WT) and IL-13 KO mice. Mean \pm s.e.m., $n=2-8 /$ group/experiment, two pooled experiments. CT, cholera toxin; FMO, fluorescence minus one; PMA, phorbol-12-myristate-13-acetate; TCR, T-cell receptor. ${ }^{*} P<0.05$ vs $-\alpha C D 90.2$.

responses, the original source of IL-4 in the initiation of type 2 responses to food allergens remains unknown. Herein, we report that the development of type 2 immunity during PN allergic sensitization is independent of $\gamma \delta$ T cells, NKT cells, or ILC2s and is, instead, driven by T helper cell-intrinsic IL-4 under the control of OX40L.

A large body of literature supports the notion that non- $T$ helper cell derived IL-4 is required to initiate Th2 responses. ${ }^{4,5}$ Indeed, classical in vitro polarization of naïve $\mathrm{T}$ cells to Th2 cells requires supplementation with exogenous IL-4 in addition to TCR stimulation. Thus, a popular paradigm is that innate cell types capable of rapidly producing IL-4 after stimulation have critical roles in driving Th2 polarization in vivo. Such a function has been ascribed to mast cells, basophils, NKT cells, and $\gamma \delta \mathrm{T}$ cells. Notably, we have previously shown that mast cells, B cells, and basophils are not required to induce intestinal Th2 immunity to $\mathrm{PN}^{17,20}$

The role of NKT cells in the initiation of food allergy has not been examined previously, but these cells have been shown to be activated by food lipids. ${ }^{15}$ Further, NKT cells can produce type 2 cytokines and, thus, regulate the development of Th2 immunity, in particular, in IgE production and allergic asthma. $^{13,14,26}$ Here, we found that IL-15, $\beta 2 \mathrm{~m}$, and CD1 KO mice and anti-NK1.1-treated WT mice had comparable levels of PN-specific IgE, Th2 cytokine production, and eosinophilic inflammatory responses to WT or non-depleted NKT-sufficient mice (Figure 2; Supplementary Figure S1). Collectively, our IL-15 KO, $\beta 2 \mathrm{~m} \mathrm{KO}, \mathrm{CD} 1 \mathrm{KO}$, and anti-NK1.1 data support the idea that NKT cells do not augment or inhibit Th2 responses in this system. 


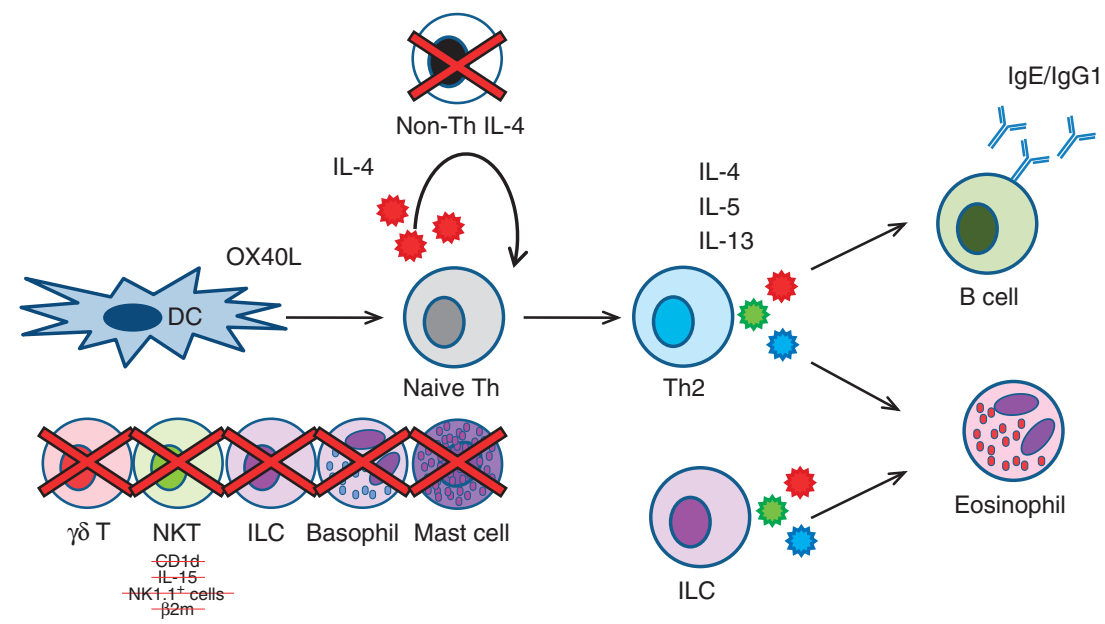

Figure 7 Model of Thelper type 2 (Th2) induction during peanut (PN) allergic sensitization. Allergen exposure at epithelial sites activates dendritic cells (DCs) to express OX40L in an interleukin-33 (IL-33)-dependent manner. ${ }^{17}$ Within the mesenteric lymph node (MLN), DC OX40L triggers naïve T cells to secrete IL-4, which then acts in an autocrine/paracrine manner to amplify and stabilize Th2 differentiation. Differentiated Th2 cells then act within lymph nodes (LNs) to help B cells class switch to IgE and IgG1 in an IL-4-dependent, IL-13-independent manner. ${ }^{17}$ Effector Th2 cells exit the lymph node to induce IL-13-dependent eosinophilia at sites of allergen exposure in cooperation with group 2 innate lymphoid cells (ILC2s). Mast cells, basophils, $\gamma \delta \mathrm{T}$ cells, natural killer-like T (NKT) cells (and IL-15, $\beta 2$ microglobulin $(\beta 2 \mathrm{~m}), \mathrm{CD} 1$, and NK1.1 ${ }^{+}$cells), ILCs and all non-Th IL-4 are dispensable to induce adaptive Th2 immunity during priming for PN allergy and anaphylaxis.

$\gamma \delta$ T cells are abundant within mucosal surfaces in both mice and humans. Bol-Schoenmakers et al. ${ }^{27}$ have recently proposed that $\gamma \delta \mathrm{T}$ cells negatively regulate the induction of PN allergy based on observations that $\mathrm{PN}+\mathrm{CT}$ exposure decreases intestinal $\gamma \delta$ T-cell proportions, and administration of anti$\mathrm{TCR} \gamma \delta$ clone UC7-13D5 during $\mathrm{PN}+\mathrm{CT}$ sensitization resulted in increased serum PN-IgE, but not PN-IgG1 or PN-IgG2a levels. Unfortunately, other biological or clinical readouts (e.g., anaphylaxis) were not examined in these studies. In contrast, here, we found that TCR $\delta$ KO mice sensitized to PN and their WT controls mount not only comparable PN-specific IgG1 and IgE but also similar clinical anaphylactic (hypothermia, hemoconcentration, clinical signs), inflammatory and cytokine responses (Figure 3). We obtained similar results to our KO data using UC7-13D5 antibody throughout our PN sensitization protocol (Supplementary Figure S2) or the one described by Bol-Schoenmakers et al. Altogether, our observations lead us to propose that $\gamma \delta \mathrm{T}$ cells do not significantly regulate the initiation of PN allergy in this system. In line with this, $\mathrm{Kit}^{\mathrm{Wv} / \mathrm{v}}$ mice are also deficient in intestinal $\gamma \delta \mathrm{T}$ cells, ${ }^{28}$ and we have previously shown that these mice mount robust Th2 immunity including IgE production, inflammation, and cytokines. ${ }^{18,20}$ Other studies using adoptive transfer, genetic and antibody-based approaches have reported that $\gamma \delta \mathrm{T}$ cells may be required to promote Th2 responses and/or IgE production. ${ }^{8,9}$ For example, $\gamma \delta$ T cell-deficient mice showed decreased levels of serum ovalbumin-specific IgE and IgG1 antibodies after intraperitoneal immunization with ovalbumin, which were restored with administration of IL- $4 .{ }^{9}$ However, we could not find any impact of TCR $\delta$ deficiency or anti-TCR $\gamma \delta$ treatment on multiple immunological, clinical, and physiological parameters of Th2 immunity and $\mathrm{PN}$-induced anaphylaxis.
ILCs are a novel cell type that lack lineage markers (non-B, non-T, CD11c- $\mathrm{CD}^{-} 1 \mathrm{~b}^{-}$), express c-Kit, Sca-1, and $\mathrm{CD}^{7} 0^{7,25}$ and have been reported to be increased in the intestine and draining LN during PN sensitization. ${ }^{17}$ CD90 is a T-cell maker with genetic polymorphisms across mouse strains which allows for adoptive transfer and tracking studies. Although CD90 is also expressed by non-T cells such as stromal cells, generation of CD90 disparate chimeras is a recently established method to selectively deplete ILCs. ${ }^{24}$ Indeed, it has been in such a system that ILCs have been shown to regulate T-cell responses. ${ }^{24}$ Although ILC2s are known to be important to effecting anti-helminth immunity ${ }^{25}$ the role of ILC2s in regulating the initiation of Th2 responses remains unclear. The main cytokines produced by these cells are IL-13 and IL-5, and IL-4. ${ }^{7,25}$ Herein, we generated CD90-disparate chimeras and found that ILCs are not required to initiate Th2 immunity, but are important for effecting type 2 inflammatory responses, including the accumulation of eosinophils, through IL-5 and IL-13 production (Figure 6). Indeed, ILC2s produced IL-5 and IL-13, and loss of ILC2s led to a loss of peritoneal IL-5 and IL-13. Consistent with this, a relative phenocopying of PN allergic responses was observed in both ILC2-deficient and IL-13 deficient mice: unimpaired Th2 priming, but defective effector inflammatory responses. Although multiple non-Th cell types are potentially IL- 4 competentNKT cells, $\gamma \delta \mathrm{T}$ cells, ILCs, basophils, mast cells, and eosinophils ${ }^{29}$ (Supplementary Figure S4a)-we conclusively show that all non-CD4 ${ }^{+}$sources of IL- 4 are not critical for the initiation of Th2 responses in vivo (Figure 4). Hence, IL-4 derived solely from $\mathrm{CD} 4{ }^{+} \mathrm{T}$ helper cells is fully sufficient to induce clinically and biologically meaningful Th2-polarized allergic responses in vivo. 
Competing and dichotomous models of Th2 differentiation argue for IL-4-dependent vs. IL-4-independent pathways. ${ }^{21,30}$ The latter has been proposed to be driven by costimulation, and the former, by IL-4R signaling. ${ }^{16,31}$ The outcome of either pathway is upregulation of lineage determining factors such as GATA3, leading to initiation of Th2 phenotype differentiation and secretion of signature cytokines such as IL-4, IL-5, and IL13. In response to $\mathrm{PN}$ allergic sensitization we not only found that $\mathrm{CD}^{+}{ }^{+} \mathrm{T}$ helper cell-intrinsic IL-4, alone, drove this process, but also that in vivo IL-4 upregulation required OX40L costimulation (Figure 5). Hence, we provide evidence for a pathway that requires OX40L costimulation for IL-4 production, yet also IL-4 for complete Th2 development. OX40L has been shown to regulate IL-4 secretion early after in vitro $\mathrm{T}$-cell stimulation or primary helminth infection, ${ }^{32,33}$ potentially through NFAT2, which is also known as NFATc1. ${ }^{31}$ Independent from this, multiple reports have shown that early, low levels of IL-4 secretion from $\mathrm{CD}^{+}{ }^{+} \mathrm{T}$ cells can be independent of IL-4, IL-4R $\alpha$, or STAT6, but that intact IL-4 signaling is required for a maximal, terminal Th2 effector profile..$^{21,30,34,35}$ Thus, impairments in Th2 development due to IL-4 signaling deficiency typically do not become apparent until later time points, such as in effector tissue responses or secondary infections. Altogether, these and our data support an at least two-step model of IL-4 production, whereby naïve $\mathrm{CD} 4{ }^{+} \mathrm{T}$ cells initially produce IL-4 in response to costimulation (such as through OX40-OX40L), and that subsequent autocrine/paracrine signaling of $\mathrm{CD} 4{ }^{+} \mathrm{T}$ helper cell-intrinsic IL-4 amplifies and stabilizes the Th2 state (Figure 7), in a manner somewhat analogous to IL-23-mediated stabilization of Th17 differentiation, or IFN- $\gamma$ during Th1 differentiation. ${ }^{6}$ Thus, DC OX40L-mediated triggering of IL- 4 production from naïve $\mathrm{T}$ cells initiates a self-driven Th2 differentiation/ amplification pathway that leads to the development of PN allergy and anaphylaxis independent of innate lymphocytes.

\section{METHODS}

Mice. Age, sex, and strain-matched controls were used in all experiments. CD $45.1^{+}$mice (B6.SJL-Ptprc ${ }^{a} P e p c^{b} /$ BoyJ), IL-4 KO $\left(\mathrm{B} 6.129 \mathrm{P} 2-\mathrm{Il}^{\text {tm1Cgn }} / \mathrm{J}\right)$, TCR $\delta$ KO (B6.129P2-Tcrd $\left.{ }^{\text {tm1Mom}} / \mathrm{J}\right)$, TCR $\beta$ KO (B6.129P2-Tcrb $\left.{ }^{\text {tm1Mom }} / \mathrm{J}\right)$, CD90.1 ${ }^{+}$mice (B6.PL-Thy1 $\left.{ }^{a} / \mathrm{CyJ}\right)$, $\mathrm{CD} 1 \mathrm{KO}\left(\mathrm{B} 6.129 \mathrm{~S} 6-\mathrm{Cd} 1 \mathrm{~d} 1 / \mathrm{Cd} 1 \mathrm{~d} 2^{\text {tm ISpb }} / \mathrm{J}\right)$, and 4get (C.129-Il4 $\left.{ }^{\mathrm{tm} 1 \mathrm{Lky}} / \mathrm{J}\right)$ mice were from JAX Laboratories (Bar Harbor, ME). $\beta 2 \mathrm{~m} \mathrm{KO}$ (B6.129$\left.B 2 \mathrm{~m}^{\text {tmlJae }}\right)$ mice were from Taconic (Germantown, NY). IL-15 KO (C57BL/6NTac-IL15 ${ }^{\text {tm1Imx }}$ ) and IL-13 KO (C.129P2-Il13 ${ }^{\text {tm2Anjm }}$ ) mice were bred in-house. All procedures were approved by the McMaster University Research Ethics Board.

PN allergy model including antibody administration. Intragastric gavage (Delvo, Biel/Bienne, Switzerland) of PN (Kraft, Northfield, IL) with CT (List Biologicals, Campbell, CA) was performed weekly for 4 weeks, with challenge by crude PN extract (Greer, Lenoir, NC) 1 week later. Serum was collected $24 \mathrm{~h}$ before challenge and analyzed for total or PN-specific immunoglobulins. ${ }^{17}$ In some experiments, anti-TCR $\gamma \delta$ antibody UC7-13D5 (BioXCell, West Lebanon, NH), or anti-OX40L (R\&D Systems, Minneapolis, MN) was given $24 \mathrm{~h}$ before each gavage. For ILC depletion, $1 \mathrm{mg}$ anti-Thy1.2 (30H12, made in-house) was administered to chimeric mice every 3-4 days beginning 1 week before the first gavage. In all, $450 \mu \mathrm{g}$ anti-NK1.1 (PK136 (ref. 36) hybridoma HB191 from HTCC, made in-house), was given 3 days and 1 day before the first gavage, and every 3 days thereafter until the last gavage. In some anti-TCR $\gamma \delta$ antibody UC7-13D5 experiments, the protocol described by Bol-Schoenmakers et al. ${ }^{27}$ was employed. Temperature was assessed by rectal probe, and hematocrit by centrifuging anticoagulated blood at 40' after challenge. Peritoneal lavage was performed with PBS-EDTA. Total cells were counted using Turks and hemocytometer. Eosinophils were quantified using flow cytometry as $\mathrm{F} 4 / 80^{\mathrm{lo}}$, Siglec- $\mathrm{F}^{+}$cells or manual differential cell counting of at least 500 cells from cytospins (Thermo Shandon, Waltham, MA) stained using Hema 3 (Fisher Scientific, Hampton, NH), with good agreement between both methods.

Cytokine production. Spleens were collected $72 \mathrm{~h}$ after challenge and 800000 live splenocytes were cultured for $120 \mathrm{~h}$ in media alone or supplemented with $250 \mu \mathrm{g} \mathrm{ml}^{-1}$ crude PN extract and cytokines in supernatants were quantified using Luminex (Millipore, Temeoula, CA) or ELISA (R\&D). Peritoneal lavage cell-free supernatants were analyzed using ELISA (R\&D).

Adoptive transfer and chimera generation. CD90 or CD45 chimeras were generated by isolating CD ${ }^{+} \mathrm{T}$ cells from pooled spleens and LNs from Thy $1.1^{+}$or CD $45.1^{+}$mice by magnetic-activated cell sorting (Stemcell, Vancouver, BC) to $>95 \%$ purity and transferring $10^{7}$ cells intravenously to Thy1.2 ${ }^{+}$TCR $\beta$ KO or $5.5 \mathrm{~Gy}$ ( $\mathrm{Cs}^{137}$ source) sublethally irradiated CD $45.2^{+}$WT or IL- $4 \mathrm{KO}$ mice, respectively. Sensitization was initiated 2 weeks post irradiation.

Flow cytometry. In all assays, cells were incubated with anti-Fc $\gamma$ RII/ IIIb before incubation with fluorochrome-conjugated antibodies, dead cells excluded by propidium iodide (Sigma, St Louis, MO) uptake or fixable Viability Dye eFluor780 (eBioscience, San Diego, CA), gated on singlets, and fluorescence minus one controls were used for gating. Whole blood was EDTA anticoagulated and lysed with ammoniumchloride-potassium before staining. Antibodies were from eBioscience, BD Biosciences (San Jose, CA), or Biolegend (San Diego, CA): CD3FITC, Pacific Blue, Brilliant Violet 421 or BV785; CD4-APC, eFluor605 or eFluor650; CD19 PE-Cy5; CD45.1-eFluor 605; CD45.2Pacific Blue; MHC II-eFluor 650; F4/80-eFluor 450 or Pacific Blue; Siglec-F-PE; NK1.1-PE-Cy7 or BV510; CD49b (DX5)-PE or PE-Cy7; TCR $\beta$-FITC or Pacific Blue; TCR $\gamma \delta$-PE; Sca-1-Alexa Fluor 700; CD44V500 or Alexa Fluor 700; CD62L-APC-eFluor 780; B220-eFluor650 or V500; CD90.1-Alexa Fluor 700 or eFluor605; CD90.2-APC-eFluor780, PE-Cy5 or FITC, CD11c-PerCP-Cy5.5 or PE-Cy5, CD11b-PerCPCy5.5 or PE-Cy5, TER119-APC, IL-7R $\alpha$-PE-Cy7, IL-5-PE, c-Kit-APC or BV605, IL-13-Alexa Fluor 488, CD25-PerCP-Cy5.5. ST2-FITC or -biotin was from MD Bioproducts (Zürich, Switzerland). For intracellular cytokine staining, cells were activated with phorbol12 -myristate-13-acetate $\left(50 \mathrm{ng} \mathrm{ml}^{-1}\right)$ and ionomycin $\left(750 \mathrm{ng} \mathrm{ml}^{-1}\right.$ ) (both from Sigma) with Brefeldin A (eBioscience, $10 \mu \mathrm{g} \mathrm{ml}^{-1}$ ) for $6 \mathrm{~h}$ before fixation/permeabilization (BD Biosciences) and analysis. Data were acquired on an LSR II (BD Biosciences) and analyzed using FlowJo (Treestar, Ashland, OR).

Statistics. Comparisons were made using unpaired $t$-tests or one or two-way ANOVA. Repeated measures were used for temperature data. $P<0.05$ was considered as statistically significant. The number of experiments performed is indicated at the end of each figure legend.

SUPPLEMENTARY MATERIAL is linked to the online version of the paper at http://www.nature.com/mi

\section{ACKNOWLEDGMENTS}

We thank Dr Jennifer Basset for assistance with irradiation and T-cell transfer, Juliana Xie for technical assistance and AllerGen NCE for their support of this, and Cassandra Colbert for additional help. We are grateful to Dr Dawn Bowdish and Dr Kathy McCoy for insightful comments. We thank Dr Andrew N.J. McKenzie for IL-13 KO mice. 


\section{DISCLOSURE}

A.A.H. and R.K. are employees of Medlmmune LLC. D.K.C. is a CIHR Vanier Scholar. A.L.G. was supported by a Fundación Caja Madrid doctoral scholarship (Spain). H.V. was supported by an Ontario Graduate Scholarship. M.J. holds a Senior Canada Research Chair in Immunobiology of Respiratory Diseases and Allergy. This work was funded by grants from Anaphylaxis Canada and Medlmmune LLC.

(c) 2014 Society for Mucosal Immunology

\section{REFERENCES}

1. Chafen, J.J. et al. Diagnosing and managing common food allergies: a systematic review. JAMA 303, 1848-1856 (2010).

2. Rona, R.J. et al. The prevalence of food allergy: a meta-analysis. J. Allergy Clin. Immunol. 120, 638-646 (2007).

3. Burks, A.W. Peanut allergy. Lancet 371, 1538-1546 (2008).

4. Paul, W.E. What determines Th2 differentiation, in vitro and in vivo? Immunol. Cell Biol. 88, 236-239 (2010).

5. Paul, W.E. \& Zhu, J. How are $T(H) 2$-type immune responses initiated and amplified?. Nat. Rev. Immunol. 10, 225-235 (2010).

6. Zhu, J., Yamane, H. \& Paul, W.E. Differentiation of effector CD4 T cell populations (*). Annu. Rev. Immunol. 28, 445-489 (2010).

7. Licona-Limon, P., Kim, L.K., Palm, N.W. \& Flavell, R.A. TH2, allergy and group 2 innate lymphoid cells. Nat. Immunol. 14, 536-542 (2013).

8. Hayday, A.C. Gammadelta T cells and the lymphoid stress-surveillance response. Immunity 31, 184-196 (2009).

9. Zuany-Amorim, C., Ruffie, C., Haile, S., Vargaftig, B.B., Pereira, P. \& Pretolani, M. Requirement for gammadelta $T$ cells in allergic airway inflammation. Science 280, 1265-1267 (1998).

10. Bendelac, A., Savage, P.B. \& Teyton, L. The biology of NKT cells. Annu. Rev. Immunol. 25, 297-336 (2007).

11. Gordy, L.E. et al. IL-15 regulates homeostasis and terminal maturation of NKT cells. J. Immunol. 187, 6335-6345 (2011).

12. Ma, A., Koka, R. \& Burkett, P. Diverse functions of IL-2, IL-15, and IL-7 in lymphoid homeostasis. Annu. Rev. Immunol 24, 657-679 (2006).

13. Akbari, O. et al. Essential role of NKT cells producing IL-4 and IL-13 in the development of allergen-induced airway hyperreactivity. Nat. Med. 9 , 582-588 (2003).

14. Lisbonne, M. et al. Cutting edge: invariant $V$ alpha 14 NKT cells are required for allergen-induced airway inflammation and hyperreactivity in an experimental asthma model. J. Immunol. 171, 1637-1641 (2003).

15. Jyonouchi, S. et al. Invariant natural killer T cells from children with versus without food allergy exhibit differential responsiveness to milk-derived sphingomyelin. J. Allergy Clin. Immunol. 128, 102-109 e113 (2011).

16. van Panhuys, N. et al. In vivo studies fail to reveal a role for IL-4 or STAT6 signaling in Th2 lymphocyte differentiation. Proc. Natl. Acad. Sci. USA 105 12423-12428 (2008).

17. Chu, D.K. et al. IL-33, but not thymic stromal lymphopoietin or IL-25, is central to mite and peanut allergic sensitization. J. Allergy Clin. Immunol. 131, 187-200 e188 (2013).

18. Arias, $\mathrm{K}$. et al. Distinct immune effector pathways contribute to the full expression of peanut-induced anaphylactic reactions in mice. J. Allergy Clin. Immunol. 127, 1552-1561 e1551 (2011).
19. Arias, K. et al. Concurrent blockade of platelet-activating factor and histamine prevents life-threatening peanut-induced anaphylactic reactions. J. Allergy Clin. Immunol. 124, 307-314. 314.e1-2 (2009).

20. Sun, J. et al. Impact of CD40 ligand, B cells, and mast cells in peanut-induced anaphylactic responses. J. Immunol. 179, 6696-6703 (2007).

21. Mohrs, M., Shinkai, K., Mohrs, K. \& Locksley, R.M. Analysis of type 2 immunity in vivo with a bicistronic IL-4 reporter. Immunity 15, 303-311 (2001).

22. Klein Wolterink, R.G. et al. Pulmonary innate lymphoid cells are major producers of IL-5 and IL-13 in murine models of allergic asthma. Eur. J. Immunol. 42, 1106-1116 (2012).

23. Price, A.E. et al. Systemically dispersed innate IL-13-expressing cells in type 2 immunity. Proc. Natl. Acad. Sci. USA 107, 11489-11494 (2010).

24. Hepworth, M.R. et al. Innate lymphoid cells regulate CD4 + T-cell responses to intestinal commensal bacteria. Nature 498, 113-117 (2013).

25. Spits, H. \& Cupedo, T. Innate lymphoid cells: emerging insights in development, lineage relationships, and function. Annu. Rev. Immunol. 30, 647-675 (2012).

26. Yoshimoto, T., Bendelac, A., Watson, C., Hu-Li, J. \& Paul, W.E. Role of NK1.1 + T cells in a Th2 response and in immunoglobulin E production. Science 270, 1845-1847 (1995).

27. Bol-Schoenmakers, M. et al. Regulation by intestinal gammadelta T cells during establishment of food allergic sensitization in mice. Allergy 66, 331340 (2011).

28. Grimbaldeston, M.A., Chen, C.C., Piliponsky, A.M., Tsai, M., Tam, S.Y. \& Galli, S.J. Mast cell-deficient W-sash c-kit mutant Kit W-sh/W-sh mice as a model for investigating mast cell biology in vivo. Am. J. Pathol. 167, 835848 (2005).

29. Gessner, A., Mohrs, K. \& Mohrs, M. Mast cells, basophils, and eosinophils acquire constitutive IL-4 and IL-13 transcripts during lineage differentiation that are sufficient for rapid cytokine production. J. Immunol. 174, 10631072 (2005).

30. Finkelman, F.D. et al. Stat6 regulation of in vivo IL-4 responses. J. Immunol. 164, 2303-2310 (2000).

31. So, T., Song, J., Sugie, K., Altman, A. \& Croft, M. Signals from OX40 regulate nuclear factor of activated T cells $\mathrm{c} 1$ and $\mathrm{T}$ cell helper 2 lineage commitment. Proc. Natl. Acad. Sci. USA 103, 3740-3745 (2006).

32. Ekkens, M.J. et al. The role of OX40 ligand interactions in the development of the Th2 response to the gastrointestinal nematode parasite Heligmosomoides polygyrus. J. Immunol. 170, 384-393 (2003).

33. Ohshima, Y. et al. OX40 costimulation enhances interleukin-4 (IL-4) expression at priming and promotes the differentiation of naive human CD4(+) T cells into high IL-4-producing effectors. Blood 92, 3338-3345 (1998).

34. Jankovic, D., Kullberg, M.C., Noben-Trauth, N., Caspar, P., Paul, W.E. \& Sher, A. Single cell analysis reveals that IL-4 receptor/Stat6 signaling is not required for the in vivo or in vitro development of CD4 + lymphocytes with a Th2 cytokine profile. J. Immunol. 164, 3047-3055 (2000).

35. Voehringer, D., Shinkai, K. \& Locksley, R.M. Type 2 immunity reflects orchestrated recruitment of cells committed to IL-4 production. Immunity 20, 267-277 (2004).

36. Koo, G.C., Dumont, F.J., Tutt, M., Hackett, J. Jr. \& Kumar, V. The NK1.1(-) mouse: a model to study differentiation of murine NK cells. J. Immunol. (Baltimore, Md: 1950) 137, 3742-3747 (1986). 